\title{
Comparison of experimental and predicted TCP solvus temperatures in Ni-base superalloys
}

\author{
A.S. Wilson ${ }^{\mathrm{a}^{*}}$, M.C. Hardy ${ }^{\mathrm{b}}$, H. J. Stone ${ }^{\mathrm{a}}$. \\ ${ }^{\text {a }}$ Department of Materials Science and Metallurgy, University of Cambridge, Cambridge, CB3 OFS \\ ${ }^{\mathrm{b}}$ Rolls-Royce plc, PO Box 31, Derby, DE24 8BJ \\ *Corresponding author, e-mail asw33@cam.ac.uk
}

\begin{abstract}
Differential Scanning Calorimetry has been used for the first time to quantify sigma solvus temperatures in a series of model nickel-based alloys. These data were compared with thermodynamic predictions of the sigma solvus temperatures made using Thermocalc software to assess the fidelity of the available databases. It was found that the expected trend of increasing solvus temperature with increasing Mo content is correctly predicted, but that the solvus temperatures themselves are significantly under-predicted. Due to the important role that topologically close-packed phases play in limiting the service life of nickel-based superalloys, this has important implications for the use of sigma solvus temperature predictions in the alloy design process.
\end{abstract}

\section{$\underline{\text { Keywords }}$}

Nickel superalloys, Topologically close-packed phases, Sigma, Differential Scanning Calorimetry, Solvus temperatures

\section{Introduction}

Nickel-base superalloys are used for the hot section components of turbine engines, such as turbine discs and blades. International environmental regulations for the reduction of emissions from civil aircraft [1,2] require the next generation of engines to be more efficient, necessitating their operation at temperatures and stresses beyond the capabilities of current superalloys. As a result, new alloys must be designed that can withstand these increasingly demanding conditions. Optimising these new alloy compositions requires balancing directly competing requirements. For example, increased $\mathrm{Cr}$ contents are needed to provide environmental resistance and increased concentrations of other refractory metals to improve solid solution strengthening. However, these elements compromise the alloy's long-term microstructural stability by promoting the formation of brittle intermetallic phases, known as topologically closepacked (TCP) phases, which are deleterious to alloy performance. High $\gamma^{\prime}$ volume fractions, which are needed to provide high-temperature strength, exacerbate the problem by increasing the concentration of these elements in the $\gamma$ phase. Therefore, an understanding of alloy susceptibility to TCP formation is vital in the design of new alloy compositions.

The experimental assessment of an alloy's thermal stability requires analysis of samples after multiple long-term thermal exposures of different durations and temperatures. This is a time and cost-intensive process. As a result, thermodynamic predictions of TCP solvus temperatures and phase fractions are regularly used as tools 
to assess thermal stability during the early stages of the design of new alloys. It is therefore key to the success and efficiency of the design process that we understand the accuracy of these predictions.

A significant amount of experimental data for phase fractions of TCP phases formed under various conditions can be found in the literature, along with some comparisons between these experimental data and thermodynamic predictions for the phase fractions [3-5]. However, scant attention has been paid to the assessment of the accuracy of TCP solvus temperature predictions, despite the fact that these are also commonly used to compare alloy performance during design. These two quantities are inextricably linked since a higher solvus temperature implies a higher driving force to form that phase at a given temperature and thus, disregarding kinetic effects, one would expect the formation of a higher volume fraction of the phase in any given time period. One attempt at such an assessment of solvus temperature predictions was made by Zhao et al. [6] who compared thermodynamic predictions made using a Thermotech database for a selection of alloys with experimental data in the form of TTT diagrams, or similar, from the literature. They concluded that the predictions were not satisfactory. However, they were reliant on existing experimental data, which consisted of indirect measurements of the TCP solvus temperatures using multiple thermal exposures at different temperatures and durations from which it is difficult to extrapolate accurate solvus temperatures. The use of differential scanning calorimetry (DSC) to obtain TCP solvus temperatures has the advantage of directly measuring the solvus temperature, thus requiring only one measurement for each alloy. It therefore has the potential to be a much faster method for assessing TCP solvus temperatures.

A significant challenge to the direct measurement of TCP solvus temperatures using thermal analysis is the low volume fractions in which these phases generally exist, which make the solvus event difficult to detect. As a result, despite the widespread use of DSC to analyse nickel-based superalloys, very few instances of the detection of TCP solvus events using DSC have been reported in the literature $[7,8]$. Furthermore, no comparison of TCP solvus temperatures detected by DSC with thermodynamic predictions has been made. In order to avoid this problem, alloys must be designed that are sufficiently unstable with respect to TCP formation that a detectable quantity of TCP phases is precipitated in a reasonable amount of time.

The aim of the present work is to directly measure the TCP solvus temperatures of a series of alloys and compare these to thermodynamic predictions made using currently available software to improve our understanding of the accuracy of such predictions. Mo is known to be an effective TCP-forming element [9] and, as such, both the amount of TCP precipitation under given conditions, and the solvus temperature, are expected to increase with increasing Mo content. Therefore, a series of alloys with varying Mo contents were designed to exhibit a variation in the $\sigma$ solvus temperature allowing a comparison with the thermodynamic predictions to be made. The alloys were designed to be model disc alloy compositions containing all the major elements that such alloys require. They did not contain any $\mathrm{C}$ or $\mathrm{B}$ to avoid precipitation of carbides or borides and were designed to precipitate only one type of TCP phase so that there would not be any difficulties in distinguishing between the solvus events of different minor phases. In addition, a lack of carbides and borides means that there is no competition for TCPforming elements, leading to a higher volume fraction of TCP precipitates and thus making the TCP solvus event easier to detect. 


\section{Experimental details}

The material used in this study was produced as bars weighing $\sim 50 \mathrm{~g}$ by arc-melting. The nominal compositions of the alloys are given in Table $\mathbf{1}$ along with their actual compositions measured using electron probe microanalysis (EPMA). These alloys are nominally $\mathrm{C}$-free but a small quantity of $\mathrm{C}$ was picked up during the melting process. This was confirmed by LECO combustion-infrared analysis of the 1 at.\% Mo and 1.25 at.\% Mo alloys, which were found to contain 0.03 and 0.04 at.\% $\mathrm{C}$ respectively. Alongside four alloys containing systematically varying Mo contents, two further alloys of similar compositions were analysed (Alloys A and B) to provide additional data for comparison with solvus temperature predictions. EPMA was carried out on a Cameca SX100 instrument and the crystal set-up used was as follows: PET - Ti, LLIF Co \& W, LPET - Mo \& Nb, LIF - Ni \& Cr, LTAP - Ta \& Al. A defocused beam of 20 $\mathrm{kV}$ and $100 \mathrm{nA}$ with a diameter of $5 \mu \mathrm{m}$ was used. The counting time was 20 seconds on the peak and 10 seconds on the background for all elements, except Ti for which these were doubled, as it was the only element analysed using the PET crystal. On each specimen three $8 \times 8$ grids with $50 \mu \mathrm{m}$ spacing between points were analysed giving a total of 192 points per specimen from which the mean composition was obtained.

Differential Scanning Calorimetry (DSC) was performed using a Netzsch 404 calorimeter with a heating and cooling rate of $10^{\circ} \mathrm{C} / \mathrm{min}$ up to $1400{ }^{\circ} \mathrm{C}$ to identify the $\gamma^{\prime}$ solvus and liquidus temperatures. From these data, a super-solvus solution treatment of $1225^{\circ} \mathrm{C}$ for 48 hours was chosen to homogenise all of the alloys. The heat treatments were carried out in box furnaces in which the temperature was checked prior to heat treatment using a probe $\mathrm{N}$-type thermocouple and the material was encapsulated in glass ampoules under an argon atmosphere to prevent oxidation. Following all heat treatments the samples were bench-cooled. After completion of the solution treatment, sections of the alloys were exposed for 500 hours at $800^{\circ} \mathrm{C}$ and sections of the 1.5 at.\% Mo alloy were additionally exposed for 175 and 1000 hours. DSC was subsequently performed on the aged material and values for the solvus temperatures of both the $\sigma$ phase and the $\gamma^{\prime}$ phase were obtained for each of the alloys by fitting a sigmoid function to the relevant solvus event. In performing this fitting an endpoint must be selected on each side of the solvus event between which fitting will be carried out, as illustrated by the schematic diagram in Fig. 1. To avoid systematic errors arising as a result of manual selection of the endpoints, multiple automated fitting procedures were carried out with each endpoint moving between chosen initial and final positions. The distance between these positions is $\sim 15-25^{\circ} \mathrm{C}$, depending on the appearance of the thermogram for that alloy. The reported solvus temperature value is an average of the values obtained across all of these fits and the reported error for this value is a combination of the error in the fitting method, given as the standard deviation calculated across all of the previously mentioned fits, and the expected instrumental error of $\pm 2^{\circ} \mathrm{C}$.

Identification of TCP phases in the aged material was carried out by electrolytic extraction of the minor phases to concentrate them for analysis and overcome issues associated with their low volume fractions in the solid alloy. The extracted residues were then characterised using X-ray diffraction (XRD) analysis. The extractions were carried out according to ASTM standard Practice E963-95 [10] with an electrolyte of $1 \%$ tartaric acid in $10 \% \mathrm{HCl}-$ methanol and a current density of $\sim 1 \mathrm{~mA} \mathrm{~mm} \mathrm{~mm}^{-2}$ for $6-8$ hours. The residue was removed from the filter paper and deposited on a single-crystal 
Si wafer in order to prevent contamination of the data by signal from the filter paper or sample holder. XRD was peformed using a Bruker D8 DAVINCI diffractometer with a position sensitive detector to maximise the intensity of the signal obtained and the speed of data collection. A CuK $\alpha$ source was used and all scans were carried out over the $2 \theta$ range $10-120^{\circ}$ with a step size of $0.02^{\circ}$ and dwell time of 2 seconds.

Scanning electron microscopy (SEM) analysis was carried out using an FEI Nova NanoSEM with a Bruker energy dispersive X-ray (EDX) detector. Samples were mounted in conductive resin and prepared using standard metallographic preparation techniques of grinding with successively finer grades of SiC paper and then polishing down to $1 \mu \mathrm{m}$ diamond paste. A final preparation stage of 20-30 minutes of polishing with colloidal silica was used for samples to be examined using back-scattered electron (BSE) imaging. Area analysis of phase fractions was carried out using the software ImageJ [11] on BSE images. Each image was processed using a bandpass filter before thresholding to select the regions of interest for analysis. Deep-etched samples resulting from the extraction process were used to observe the three-dimensional morphology of the TCP precipitates using SEM.

All thermodynamic predictions presented here were made using the modelling software Thermocalc [12], which is based on the CALPHAD method, with two different nickel superalloy databases, TTNi8 [13] and TCNi8 [14]. Details of the temperatures at which the calculations were performed and the phases that were included are presented with the results of the modelling.

\section{Results}

\subsection{Characterisation of material after exposure for 500 hours at $800^{\circ} \mathrm{C}$ :}

The intragranular microstructures of the alloys following thermal exposure for 500 hours at $800^{\circ} \mathrm{C}$ are shown in Fig. 2. The phase that appears bright white in these backscattered electron images is a TCP phase. Two main effects of Mo content on the TCP precipitation behaviour can be seen: firstly, that increasing the Mo content increases the extent of precipitation and, secondly, that as the Mo content increases, the morphology of the TCP phase changes from solely thin plate-like precipitates to a more blocky morphology.

Fig. 3 displays the XRD patterns of the electrolytically extracted residues from the alloys in the 500 hour aged condition. The majority phase identified in these spectra is $\sigma$, confirming that the bright TCP phase seen in the previous micrographs is the $\sigma$ phase. Also identified is a small amount of $\gamma / \gamma^{\prime}$, this is nearly always observed in the extraction residue due to incomplete dissolution of the $\gamma / \gamma^{\prime}$. Finally, small quantities of two phases, TiN and $\mathrm{M}_{23} \mathrm{C}_{6}$, have been identified. The relative intensity of the peaks for these phases compared with the peaks for the other phases present was much higher in the 1 at.\% Mo alloy than in the other alloys, which can be attributed to the low $\sigma$ content in this alloy. Hence, the peaks have been labelled on this spectrum rather than on the 1.75 at.\% Mo spectrum as for the other phases. TiN was found in all of the material and is a contaminant phase resulting from the arc-melting process. 
Fig. 4 shows the results of image analysis, which illustrate the increase in the area fraction of $\sigma$ phase with Mo content in the intragranular regions. The numbers given for the area fraction should not be taken as absolute due to the difficulties associated with accurate thresholding for image analysis of the $\sigma$ phase, and the use of band-pass filtering on the images. However, the images were all processed in the same way so the relative magnitudes of the values obtained are correct, allowing the trend to be determined. The intergranular precipitation behaviour also changes with Mo content, as shown by the BSE images in Fig. 5. Once again, an increase in the amount of TCP precipitation with increasing Mo content can be seen but, in addition, a second grain boundary phase is present. This phase appears dark in BSE imaging and was shown to be enriched in $\mathrm{Cr}$ using EDX. These observations resulted in the identification of this phase as $\mathrm{M}_{23} \mathrm{C}_{6}$ and this was confirmed by the presence of the $\mathrm{M}_{23} \mathrm{C}_{6}$ phase in the XRD spectra in Fig. 3. This phase is present in low volume fractions, it is precipitated primarily at grain boundaries and there is a very low grain boundary density in this material as the grain size is of the order of millimetres.

The micrographs show significant $\mathrm{M}_{23} \mathrm{C}_{6}$ precipitation in the low Mo content alloys but none in the higher Mo content alloys. This observation must be treated with caution as the different grain boundaries within a sample can vary significantly in terms of the quantity of precipitation and type of precipitates present, and it is possible to find $\mathrm{M}_{23} \mathrm{C}_{6}$ precipitates in all of the alloys studied. However, these regions have been chosen as representative of the alloys because $\mathrm{M}_{23} \mathrm{C}_{6}$ is significantly more prevalent in the low Mo alloys than in those with a high Mo content. These microstructural observations are also consistent with the XRD data, which shows the peaks relating to the $\mathrm{M}_{23} \mathrm{C}_{6}$ phase increase in intensity with decreasing Mo content, Fig. 3. The other phenomenon that can be seen in these micrographs, particularly in Fig. 5c, is the precipitation of $\gamma^{\prime}$ between the $\sigma$ phase at the grain boundary due to the rejection of $\gamma^{\prime}$ forming elements by the $\sigma$ phase.

Considering the morphology of the $\sigma$ phase in further detail, Fig. 6 shows secondary electron (SE) images of the deep- etched alloy microstructure providing information about the three-dimensional morphology of the precipitates. The intragranular platelike morphology displays a distinctive triangular pattern due to its precipitation on the (111) $\gamma$ planes (Fig. 6a) and these precipitates have an internal basket-weave morphology. The grain boundaries contain such a high density of the apparently globular intergranular precipitates that an interconnected wall down the grain boundary is formed (Fig. 6b). The breakdown of the plate-like precipitate morphology is illustrated in Fig. 6c, and Fig. 6d shows preferential precipitate coarsening at the intersections between plates.

\subsection{Prediction and measurement of $\sigma$ solvus temperatures:}

Identification of the $\sigma$ solvus temperature was performed on the 1.5 at.\% Mo alloy by comparison of the DSC thermograms before and after thermal exposure for 500 hours at $800^{\circ} \mathrm{C}$, as shown in Fig. 7. One additional solvus event can be identified in the thermally exposed material when compared to the data from the same alloy in the solution-treated state prior to thermal exposure. Interrupted DSC tests were performed to confirm that this event is related to the dissolution of $\sigma$. A sample of the aged material was heated in the DSC to $1050^{\circ} \mathrm{C}$, a temperature just below the solvus event 
indicated by arrow 1, then the flow rate of argon through the furnace was increased to cool the sample quickly. A second sample was heated to $1130^{\circ} \mathrm{C}$, which is just above the solvus event and indicated by arrow 2 . The microstructures produced are shown in Fig. $7 \mathrm{~b}$ and Fig. $7 \mathrm{c}$; it can be seen that after heating to $1050^{\circ} \mathrm{C} \sigma$ is still present, whereas after heating to $1130^{\circ} \mathrm{C}$ only $\gamma / \gamma^{\prime}$ are present, confirming that this solvus event represents the dissolution of $\sigma$.

To ensure that the heating rate of the DSC experiment $\left(10^{\circ} \mathrm{C} / \mathrm{min}\right)$ did not affect the measured temperature of the solvus event, further samples of the aged material were heat treated for up to 100 hours at $1050^{\circ} \mathrm{C}$. The microstructure produced after 100 hours at $1050^{\circ} \mathrm{C}$ is shown in Fig. 8, alongside the microstructure of the DSC sample heated to this temperature for comparison. It can be seen that $\sigma$ precipitation is still present after 100 hours at this temperature confirming that this is below the $\sigma$ solvus temperature. Area analysis of the $\sigma$ phase in these two conditions gave values of $1.82 \pm$ $0.30 \%$ in the DSC sample and $1.81 \pm 0.28 \%$ in the 100 hour sample indicating that the quantity of $\sigma$ phase in the DSC sample is representative of the equilibrium microstructural condition at this temperature.

In the literature, different studies have argued that either the return to baseline temperature [15] or, alternatively, the midpoint [16] of a fitted sigmoid, are more desirable definitions of solvus temperature when compared with the results of metallographic analysis. As a result, both the value of the midpoint and the $90 \%$ value of the sigmoid are reported in this study for the $\sigma$ phase. The midpoint represents the point of maximum rate of phase dissolution whilst the $90 \%$ point corresponds well with the point of return to baseline signal at which phase dissolution is complete. The $\gamma^{\prime}$ solvus event is more sharply defined, meaning that the $99 \%$ value is more representative of the return to baseline for this phase than the $90 \%$ value.

In order to compare these measured solvus temperature values, obtained after thermal exposure for 500 hours, with thermodynamic predictions, which reflect thermodynamic equilibrium, it must be established that the duration of the exposure does not significantly alter the $\sigma$ solvus temperature. Therefore, two further thermal exposures were carried out on the 1.75 at.\% Mo alloy, of 175 hours and 1000 hours at $800^{\circ} \mathrm{C}$. The microstructures produced by these exposures, as well as the original 500 hours at $800^{\circ} \mathrm{C}$, are illustrated in Fig. 9. From these micrographs, an increase in the quantity of $\sigma$ precipitation with exposure time can be seen, as well as a change to a more blocky precipitate morphology. In addition, a variation of $\sim 5^{\circ} \mathrm{C}$ in sigmoid midpoint and $<20^{\circ} \mathrm{C}$ in the $90 \%$ value for the solvus temperature was seen from the associated DSC data. Importantly, no trend in solvus temperature with increasing exposure time could be established. Therefore, it was concluded that it is reasonable to compare these experimental data with the thermodynamic predictions, and that the comparison would not necessarily be significantly improved by thermal exposures of longer durations.

The two thermodynamic databases used to perform thermodynamic calculations, TTNi8 and TCNi8, predicted the formation of different phases at $800^{\circ} \mathrm{C}$. TTNi8 predicted $\sigma$ to be the only TCP phase to form in all of the alloys except the 1.75 at. $\%$ Mo alloy. In this alloy, small amounts of $P$ were also predicted to form at lower temperatures, but its inclusion or suspension did not affect the predicted $\sigma$ solvus temperature. Therefore, all phases were allowed during the calculations using this database. However, TCNi8 predicted the formation of other TCP phases, such as $\mu$, in 
addition to multiple $\sigma$ phases. These additional phases were not observed in these alloys experimentally and their prediction significantly reduced the predicted $\sigma$ solvus temperatures. As a result, they were suspended from the calculations.

The experimental $\sigma$ solvus results for the varying Mo series are illustrated in Fig. 10, alongside the thermodynamic predictions made for the actual compositions of the alloys measured using EPMA. In general, it can be seen that the $\sigma$ solvus temperature increases with increasing Mo content and that the predictions made using both databases correctly reproduce this trend. In addition, the overall increase in the predicted solvus temperatures across this range of Mo contents is similar to that seen for the experimental data, being $58^{\circ} \mathrm{C}$ for the experimental $90 \%$ values and $50^{\circ} \mathrm{C}$ and $68^{\circ} \mathrm{C}$ for predictions using databases TCNi8 and TTNi8 respectively. However, the thermodynamic predictions are significantly below the experimental temperatures. Although two $\sigma$ phases are predicted using the database TCNi8, whereas TTNi8 predicts just one, it can be seen that the predicted solvus temperatures for the primary $\sigma$ phase, which are displayed on this graph, are very similar in both cases.

To further illustrate the discrepancy seen in Fig. 10, a comparison of predicted and measured solvus temperatures for both $\gamma^{\prime}$ and $\sigma$ is presented in Fig. 11. Data are included for the four alloys in the varying Mo series alongside alloys $\mathrm{A}$ and $\mathrm{B}$, which have similar compositions. Data points that lie on the diagonal black line would indicate complete agreement between the experimental data and the thermodynamic predictions. The $90 \%(\sigma)$ and $99 \%\left(\gamma^{\prime}\right)$ predicted values were chosen for this comparison rather than the sigmoid midpoints because it is considered that the point of return-to-baseline is the more useful definition of solvus temperature in practice. Regardless, the midpoint and return-to-baseline values are sufficiently similar that the conclusions drawn from this comparison would remain the same whichever set of values were used. From Fig. 11a it can be seen that the predicted $\gamma^{\prime}$ values are slightly below the measured values (average difference between predicted and measured $99 \%$ values $\approx 36^{\circ} \mathrm{C}$ ). In comparison, the predicted $\sigma$ values in Fig. $11 \mathrm{~b}$ are significantly lower than the measured values (average difference between predicted and measured $90 \%$ values $\approx 158^{\circ} \mathrm{C}$ ). Fig. $11 \mathrm{c}$ shows a comparison of experimental data for the quantity of precipitation of TCP phases in various alloys taken from the literature with the predicted phase quantities calculated using Thermocalc. The experimental data included from the literature was obtained from alloys following extended periods of thermal exposure so that, despite the slow kinetics of the $\sigma$ precipitation reaction, the alloys are in a condition as close to thermodynamic equilibrium as reasonably practicable. It can be seen that the quantity of precipitation is generally overpredicted although the size of the discrepancy varies considerably between alloys.

\section{Discussion}

\subsection{Precipitation behaviour following thermal exposure:}

An increase in the Mo content of the alloy was seen to increase the quantity of $\sigma$ precipitation (Fig. 4). This is to be expected as Mo is a potent $\sigma$-forming element, as shown by the general formula for $\sigma,(\mathrm{Cr}, \mathrm{Mo})_{x}(\mathrm{Ni}, \mathrm{Co})_{y}$ [17]. However, the effect of the Mo content on the $\sigma$ morphology, as shown in Fig. 2, merits further consideration. It can be seen that the lower Mo content alloys contain solely thin plate-like precipitates, 
whereas the alloys with higher Mo contents (particularly 1.75 at.\%) contain a blocky precipitate morphology in addition to the plate-like morphology.

One proposed explanation for this difference is that the precipitates are at different stages of morphological evolution due to the differing stabilities of the alloys. The $\sigma$ precipitate morphology evolves with time, as can be seen by the change in morphology of the precipitates in the 1.75 at.\% Mo alloy with varying exposure time shown in Fig. 9. It is thought that the exposure duration required for these changes to occur is dependent on the stability of the alloy and the resultant rate of $\sigma$ precipitation. The decreased stability of the alloys with higher Mo contents means that more precipitation has occurred during the thermal exposure of 500 hours at $800^{\circ} \mathrm{C}$ performed here, so the precipitates are further through the process of morphological evolution. The evolution process is thought to be a combination of precipitate coarsening and spheroidisation, such as that seen for cementite in steels. In martensitic steel the cementite coarsens during heat treatment; initially, the precipitates have a semi-coherent interface, but as coarsening takes place the interface gradually loses coherency [18]. This increases the interfacial energy, resulting in a tendency towards spheroidisation to minimise the interfacial energy. Considering this as the possible mechanism to account for the changes seen here, it has previously been shown that $\sigma$ plates have fully coherent interfaces with the surrounding matrix. This is the case whether the matrix is the $\gamma$ phase during the early stages of $\sigma$ precipitation, or if it is the $\gamma^{\prime}$ phase once the depletion of TCP-forming elements leads to the formation of a $\gamma^{\prime}$ envelope around the $\sigma$ precipitate [19]. It is therefore possible that the coarsening of the $\sigma$ plates leads to a reduction in the coherency of the $\sigma$-matrix interface and a corresponding driving force for spheroidisation of the precipitates to minimise the interfacial energy. A similar evolution of precipitate morphology with exposure time has previously been observed for $\mu$ phase precipitates [20,21]. For example, Sugui et al. [21] observed the initial formation of thin coherent plate-like $\mu$ precipitates, which proceeded to coarsen inhomogeneously along their length and eventually separate into a series of blocky precipitates. It is therefore suggested that the reduced stability caused by a lower Mo content means that the microstructures observed in Fig. 2 for the low Mo alloys are at earlier stages of the coarsening and spheroidising process illustrated in Fig. 9 than the microstructures of the high Mo content alloys.

An interesting feature of the coarsening behaviour that was observed in the current study is the preferential coarsening to form blocky precipitates at the intersection between two $\sigma$ plates (see Fig. 6d). This has not previously been reported in the context of the evolution of precipitates of a single phase in nickel-based alloys. Although, a similar phenomenon was observed by Matuszewski et al. [22] where the primary locations for the precipitation of coarser lath-like $\mu$ phase at the expense of plate-like $\sigma$ precipitates were at the intersection of the $\sigma$ plates.

The alloys in this study are nominally $\mathrm{C}$-free, but LECO analysis confirmed the presence of small amounts of $\mathrm{C}$, which formed $\mathrm{M}_{23} \mathrm{C}_{6}$ carbides during thermal exposure. This $\mathrm{C}$ was picked up as a contaminant during the arc-melting process. The competition between the $\sigma$ phase and $\mathrm{M}_{23} \mathrm{C}_{6}$ for elements is well-established in the literature [23,24]. Therefore, the observed trend for a higher quantity of $\mathrm{M}_{23} \mathrm{C}_{6}$ precipitation at lower Mo contents can be explained by the lower quantity of $\sigma$ precipitation in these alloys. This results in the availability of more $\mathrm{Cr}$ for $\mathrm{M}_{23} \mathrm{C}_{6}$ 
formation, in addition to less competition for the grain boundaries, which are preferential sites for nucleation of both phases.

\subsection{Prediction and measurement of $\sigma$ solvus temperatures:}

Comparison of the experimental data and the thermodynamic predictions for $\sigma$ solvus temperature in these model alloys (Fig. 11b) and for the TCP phase fraction of a variety of alloys from the literature (Fig. 11c), shows that, whilst the solvus temperatures are consistently underpredicted, the quantity of precipitation is generally overpredicted. This is unexpected as a higher solvus temperature would be expected to be associated with a larger quantity of precipitation at a given temperature below the solvus and, therefore, it would be reasonable to expect both properties to be either under- or over-predicted. The discrepancies between the predicted and experimental data are caused by problems with modelling the TCP phases, which can be attributed to their complicated nature and a lack of good experimental data available for these systems. This explains why the solvus temperature predictions for the $\gamma^{\prime}$ phase (Fig. 11a) are so much more accurate than for the $\sigma$ phase (Fig. 11b), as the $\gamma^{\prime}$ phase is much better described. It is interesting to note that predictions for the $\sigma$ phase have previously been reported to be more accurate than those for other TCPs such as $\mu, R$ and $\mathrm{P}$, due to the existence of more data on the $\sigma$ phase [25]. This suggests that the predictions for other TCP phase types may be further from the experimental values than those reported for the $\sigma$ phase here.

A potential explanation for some of the under-prediction of the $\sigma$ solvus temperatures is that a 500 hour exposure may not be sufficient to achieve thermodynamic equilibrium due to kinetic considerations and that the $\sigma$ phase is actually metastable. Such metastability has been reported previously in superalloys. In the work of Zhao et al. [25], the $\sigma$ phase was deemed to be metastable in Hastelloy $X$ and served to explain the discrepancy seen between the presence of $\sigma$ after short exposure times at temperatures up to $900^{\circ} \mathrm{C}$ and a predicted solvus temperature of $841^{\circ} \mathrm{C}$. They also observed a similar phenomenon for metastable $\gamma^{\prime}$ phase in Nimonic 263.

The potential metastability of the $\sigma$ phase is also suggested by the prediction of $\mu$ phase in addition to the $\sigma$ phase using the TCNi8 database. When both phases are allowed in the calculations, the largest effect of the prediction of $\mu$ phase is seen in the 1.75 at. $\%$ alloy in which a $\mu$ solvus temperature of $1221^{\circ} \mathrm{C}$ is predicted with a corresponding decrease in the $\sigma$ solvus temperature of $\sim 170^{\circ} \mathrm{C}$. This agrees with previous reports of kinetically inhibited precipitation of $\mu$ following the precipitation of metastable $\sigma$ [26]. If the $\sigma$ in these alloys were metastable then the true $\sigma$ solvus temperature could be lower than the experimental data suggests reducing the apparent inaccuracy of the predicted solvus temperatures. However, this is considered unlikely to account for the observed discrepancy in the current investigation for various reasons. The suspension of the $\mu$ phase in the thermodynamic calculations using the TCNi8 database still results in a large under-prediction of the $\sigma$ solvus temperature. In addition, the $\mu$ phase is not predicted by the TTNi8 database. Finally, the $\mu$ phase has not been observed in the 1.75 at.\% Mo alloy after a longer thermal exposure of 1000 hours at $800^{\circ} \mathrm{C}$, thus no evidence has been found to support the theory of metastable $\sigma$ precipitation behaviour in these alloys. 


\section{Conclusions}

Microstructural analysis of a series of thermally exposed alloys with varying Mo contents has shown that increasing the Mo content causes an increase in the quantity of $\sigma$ precipitation. Two factors have been seen to affect the morphology of the $\sigma$ precipitates, causing a change from plate-like to more blocky precipitation: increasing the Mo content of the alloy for the same thermal exposure and increasing the exposure time at temperature for the same alloy. The breakdown of the precipitate morphology is thought to occur by uneven coarsening of the precipitates followed by complete dissolution of the plates between the coarsened regions. The intersection between two plates can provide a preferential site for precipitate coarsening.

It has been shown that TCP solvus temperatures can be identified and measured using DSC in suitably designed alloys, allowing comparison of solvus temperatures between alloys and providing data for the assessment of the accuracy of solvus temperature predictions. This provides information as to how far these predictions can be relied upon when using them as guidance during alloy design. Current thermodynamic predictions correctly reproduce the trend of increasing $\sigma$ solvus temperature with Mo content but severely underpredict the measured solvus temperature values.

\section{Acknowledgements}

This work was supported by the Rolls-Royce EPSRC Strategic Partnership under EP/M005607/1, EP/ H022309/1 and EP/H500375/1. The authors would like to thank S. Rhodes for assistance with sample preparation and Dr C. Hayward at the School of Geosciences, University of Edinburgh for assistance with carrying out the EPMA work in this study. 


\section{$\underline{\text { References }}$}

[1] European aeronautics: A vision for 2020, European Commission, 2001.

[2] Flightpath 2050 Europe's vision for aviation, European Commission, 2011.

[3] R.C. Reed, M.P. Jackson, Y.S. Na, Characterization and modeling of the precipitation of the sigma phase in UDIMET 720 and UDIMET 720Li, Metall and Mat Trans A. 30A (1999) 521-533.

[4] R.A. Mitchell, Development of a New Powder Processed Ni-Base Superalloy for Rotor Disc Application, University of Cambridge, 2004.

[5] E.H. Copland, N.S. Jacobson, F.J. Ritzert, Computational Thermodynamic Predict Complex Phase Equilibria Nickel-Base Superalloy Ren6 N6, NASA, $1 \mathrm{BC}$.

[6] J.C. Zhao, M.F. Henry, Reliability of current thermodynamic data in predicting phase stability of superalloys, in: The Minerals, Metals and Materials Society, 2001.

[7] I. Lopez-Galilea, C. Zenk, S. Neumeier, S. Huth, W. Theisen, M. Göken, The thermal stability of intermetallic compounds in an as-cast SX Co-base superalloy, Adv. Eng. Mater. 17 (2015) 741-747.

[8] S. Tin, T.M. Pollock, Phase instabilities and carbon additions in single-crystal nickel-base superalloys, Materials Science and Engineering: A. 348 (2003) 111-121.

[9] A.S. Wilson, Formation and effect of topologically close-packed phases in nickel-base superalloys, Mats. Sci. Tech. 33 (2016) 1108-1118.

[10] E04 Committee, Standard Practice for Electrolytic Extraction of Phases from $\mathrm{Ni}$ and Ni-Fe Base Superalloys Using a Hydrochloric-Methanol Electrolyte, ASTM International, 2010.

[11] C.A. Schneider, W.S. Rasband, K.W. Eliceiri, NIH Image to ImageJ: 25 years of image analysis, Nature Methods. 9 (2012) 671-675.

[12] J.O. Andersson, T. Helander, L. Hoglund, P.F. Shi, B. Sundman, Thermo-Calc and DICTRA, Computational tools for materials science, Calphad. 26 (2002) 273-312.

[13] Thermo-Calc Software, Thermotech Ni-based Superalloys Database version 8.0.

[14] Thermo-Calc Software, TCS Ni-based Superalloys Database version 8.1.

[15] D.L. Sponseller, Differential Thermal Analysis of Nickel-base superalloys, in: 1996: pp. 259-270.

[16] N.G. Jones, K.A. Christofidou, P.M. Mignanelli, J.P. Minshull, M.C. Hardy, H.J. Stone, Influence of elevated $\mathrm{Co}$ and $\mathrm{Ti}$ levels on polycrystalline powder processed Ni-base superalloy, Mats. Sci. Tech. 30 (2014) 1853-1861.

[17] C.T. Sims, N.S. Stoloff, W.C. Hagel, Superalloys II, John Wiley and Sons Inc, 1987.

[18] R.C. Sharma, Principles of heat treatment of steels, New Age International Limited, 2003.

[19] K. Matuszewski, Precipitation of topologically close packed phases in Ni-base superalloys - the effect of Re and Ru, FAU Erlangen, 2016.

[20] X.Z. Qin, J.T. Guo, C. Yuan, G.X. Yang, L.Z. Zhou, H.Q. Ye, $\mu$-Phase behavior in a cast Ni-base superalloy, Journal of Materials Science. 44 (2009) 4840-4847.

[21] T. Sugui, W. Minggang, L. Tang, Q. Benjiang, X. Jun, Influence of TCP phase 
and its morphology on creep properties of single crystal nickel-based superalloys, Materials Science and Engineering: A. 527 (2010) 5444-5451.

[22] K. Matuszewski, R. Rettig, R.F. Singer, The effect of Ru on precipitation of topologically close packed phases in Re-containing Ni base superalloys: Quantitative FIB-SEM investigation and 3D image modeling, MATEC Web of Conferences. 14 (2014).

[23] P.W. Keefe, S.O. Mancuso, G.E. Maurer, Effects of Heat Treatment and Chemistry on the Long-Term Phase Stability of a High Strength Nickel-Based Superalloy, in: 1992: pp. 487-496.

[24] H.-S. Lee, D.-S. Kim, K.-B. Yoo, K.-S. Song, Quantitative analysis of carbides and the sigma phase in thermally exposed GTD-111, Met. Mater. Int. 18 (2012) 287-293.

[25] J.C. Zhao, M.F. Henry, The Thermodynamic Prediction of Phase Stability in Multicomponent Superalloys, Applied Technology Modeling HighTemperature Materials. (2002) 37-41.

[26] C.M.F. Rae, R.C. Reed, The precipitation of topologically close-packed phases in rhenium-containing superalloys, Acta Materialia. 49 (2001) 4113-4125.

[27] S.E. Kim, M.P. Jackson, R.C. Reed, C. Small, A. James, N.K. Park, Quantification of the minor precipitates in UDIMET ${ }^{\mathrm{TM}}$ alloy720(LI) using electrolytic extraction and X-ray diffraction, Materials Science and Engineering: A. 245 (1998) 225-232.

[28] J.X. Yang, Q. Zheng, X.F. Sun, H.R. Guan, Z.Q. Hu, Formation of $\mu$ phase during thermal exposure and its effect on the properties of K465 superalloy, Scripta Materialia. 55 (2006) 331-334.

[29] D.W. Hunt, Stability \& mechanical properties of a nickel-base turbine disc alloy, University of Cambridge, 2001. 


\section{Figures}

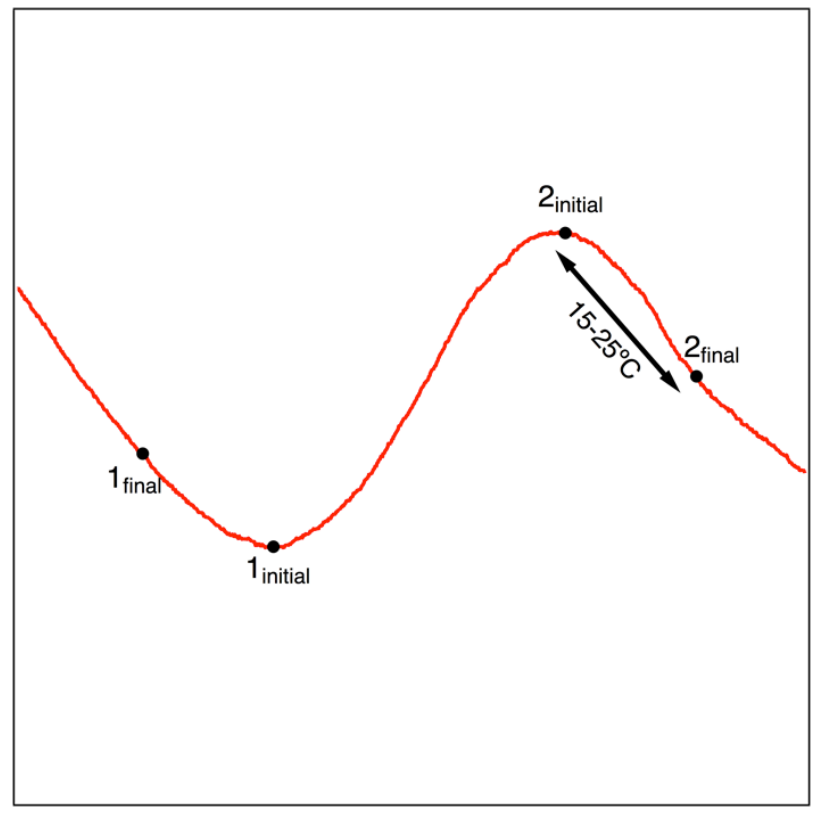

Fig. 1. Schematic diagram of solvus fitting method shown for a $\sigma$ solvus.
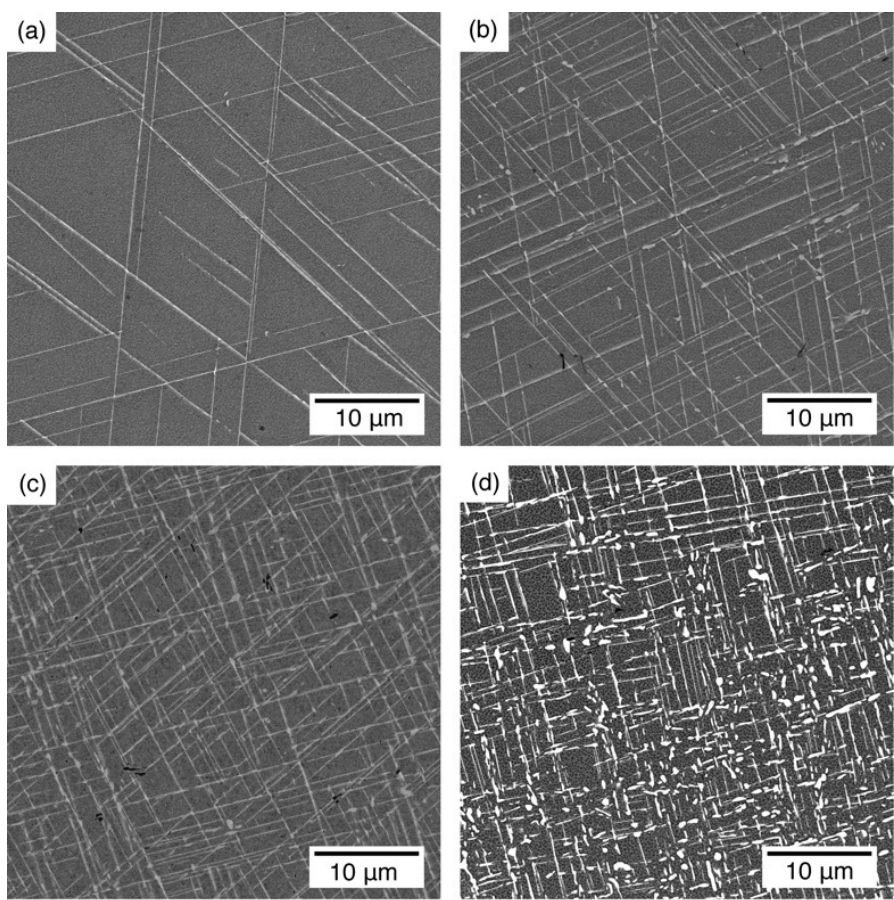

Fig. 2. BSE images of intragranular microstructures following thermal exposure for 500 hours at $800^{\circ} \mathrm{C}$ for alloys containing (a) 1 at.\% Mo, (b) 1.25 at.\% Mo, (c) 1.5 at.\% Mo and (d) 1.75 at.\% Mo. 


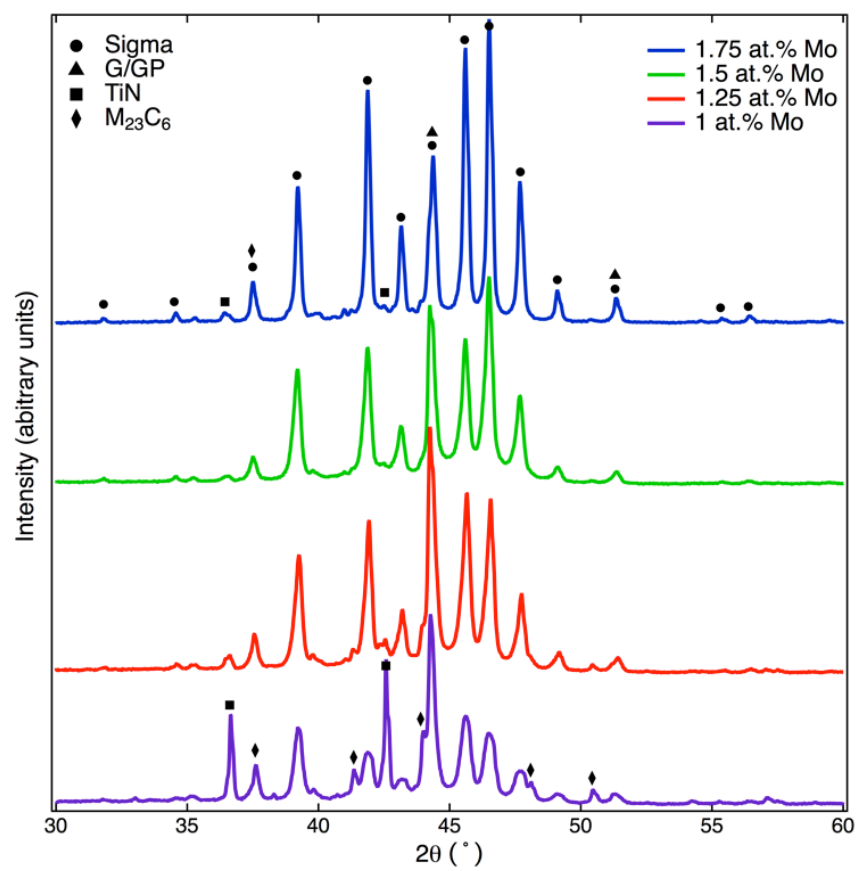

Fig. 3. XRD spectra of phase extractions from alloys following thermal exposure for 500 hours at $800^{\circ} \mathrm{C}$.

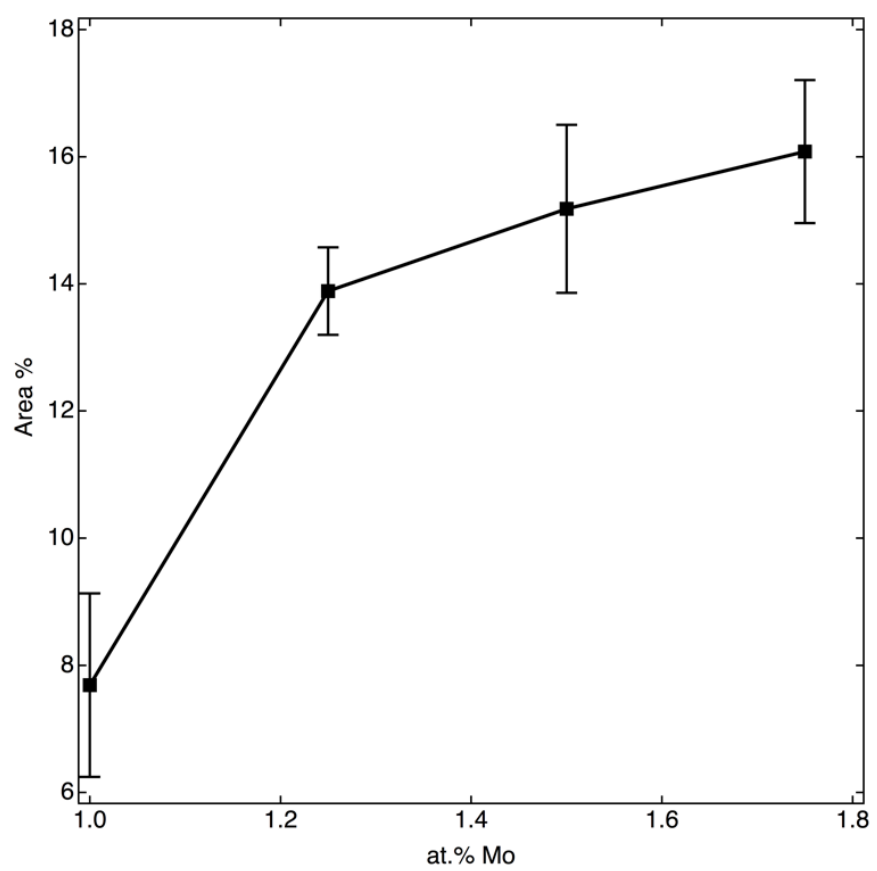

Fig. 4. Image analysis results for the effect of Mo on the area fraction of $\sigma$ in the intragranular regions following thermal exposure for 500 hours at $800^{\circ} \mathrm{C}$. 

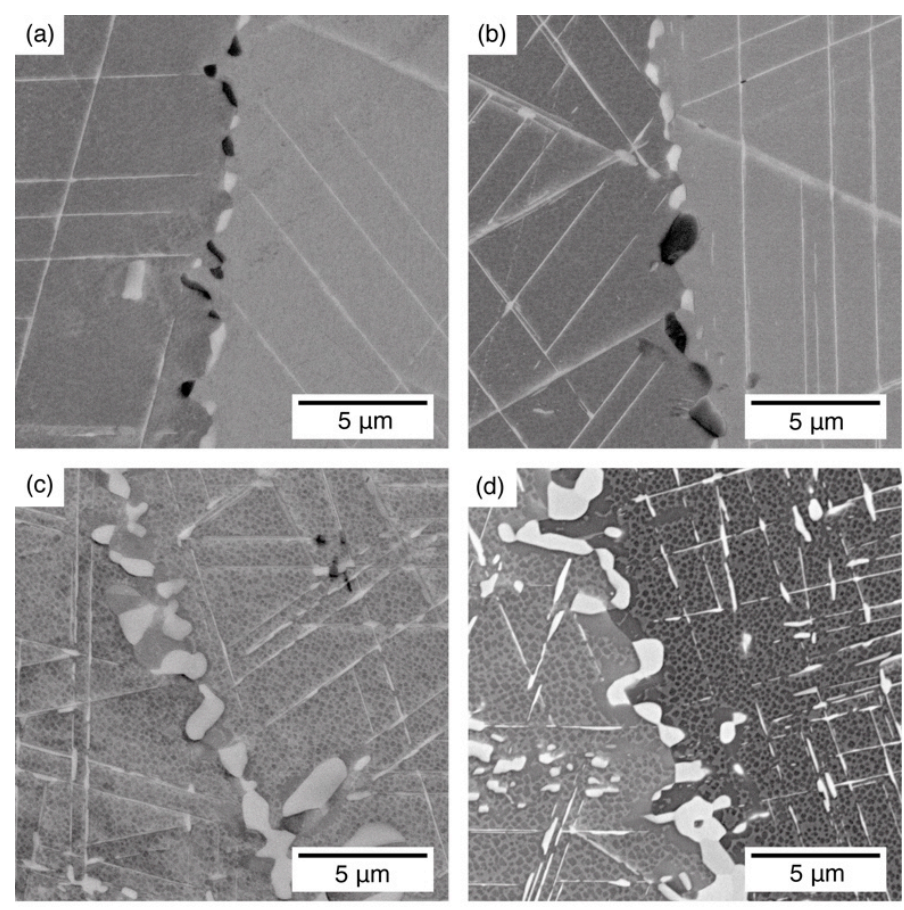

Fig. 5. BSE images of grain boundary microstructures following thermal exposure for 500 hours at $800^{\circ} \mathrm{C}$ for alloys containing (a) 1 at.\% Mo, (b) 1.25 at.\% Mo, (c) 1.5 at.\% Mo and (d) 1.75 at.\% Mo.
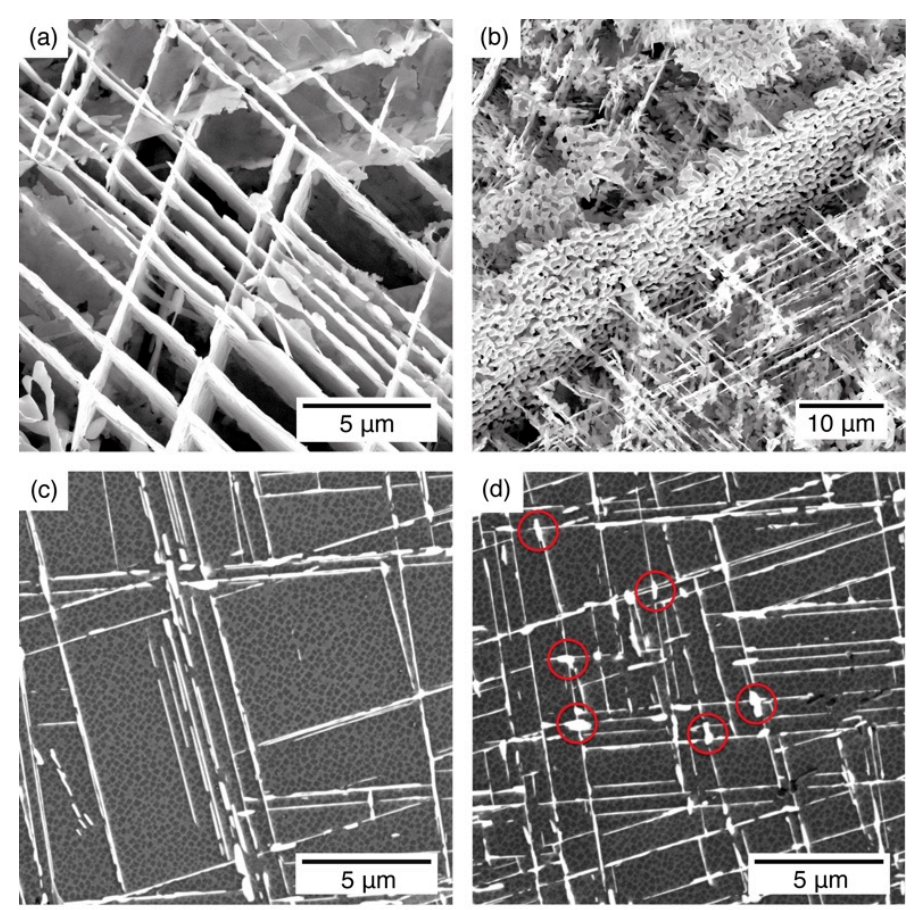

Fig. 6. SE images of deep-etched samples following thermal exposure at $800^{\circ} \mathrm{C}$ showing (a) intragranular TCP morphology in 1.5 at.\% Mo alloy and (b) intergranular TCP morphology in 1.75 at.\% Mo alloy. BSE images of $\sigma$ precipitate morphologies in 1.5 at.\% Mo alloy after thermal exposure for 500 hours at $800^{\circ} \mathrm{C}$ illustrating (c) breakdown of the plate-like precipitate morphology and (d) coarsening and growth of blocky precipitates at precipitate intersections (examples highlighted with red circles). 

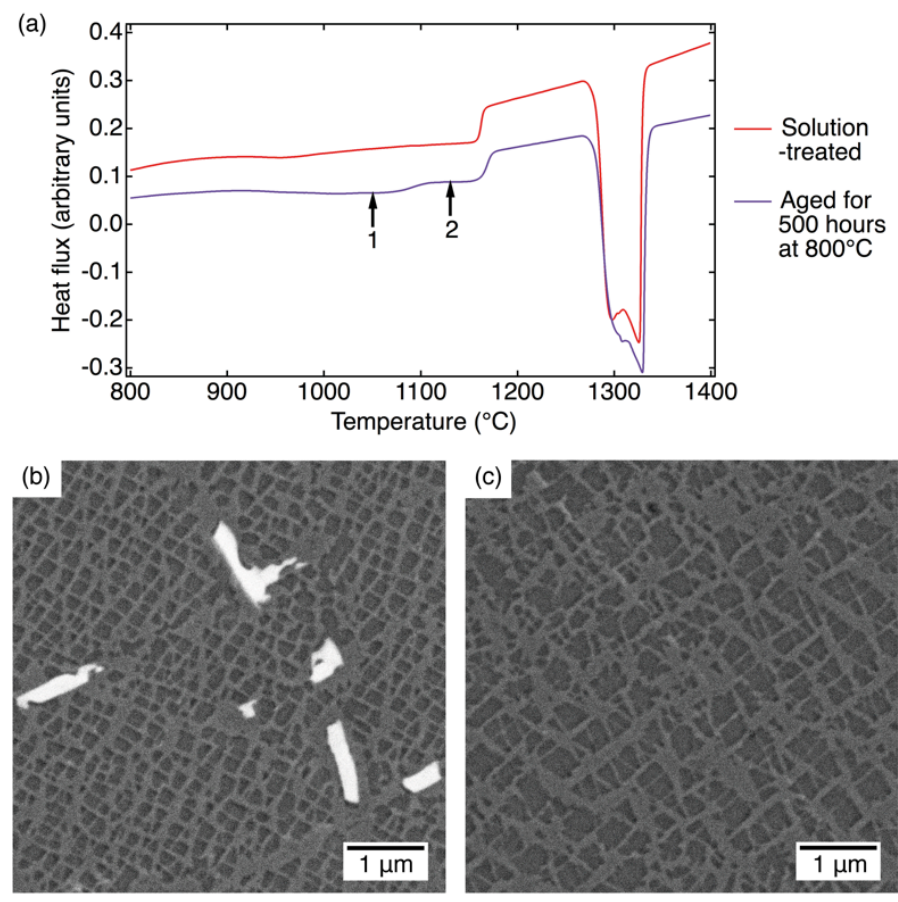

Fig. 7. Identification of $\sigma$ solvus temperature in 1.5 at.\% Mo alloy using DSC. (a) Comparison of DSC thermograms, (b) microstructure of aged material following heating to $1050^{\circ} \mathrm{C}$ (indicated by arrow 1) and (c) microstructure of aged material following heating to $1130^{\circ} \mathrm{C}$ (indicated by arrow 2 ).
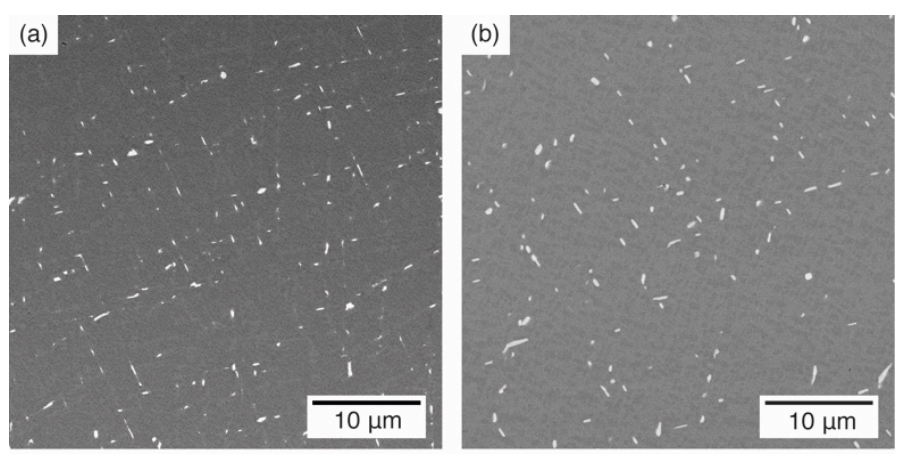

Fig. 8 BSE images of microstructure of 1.5 at.\% Mo alloy after ageing for 500 hours at $800^{\circ} \mathrm{C}$ followed by (a) heating to $1050^{\circ} \mathrm{C}$ in DSC and (b) ageing for 100 hours at $1050^{\circ} \mathrm{C}$ in a furnace. 

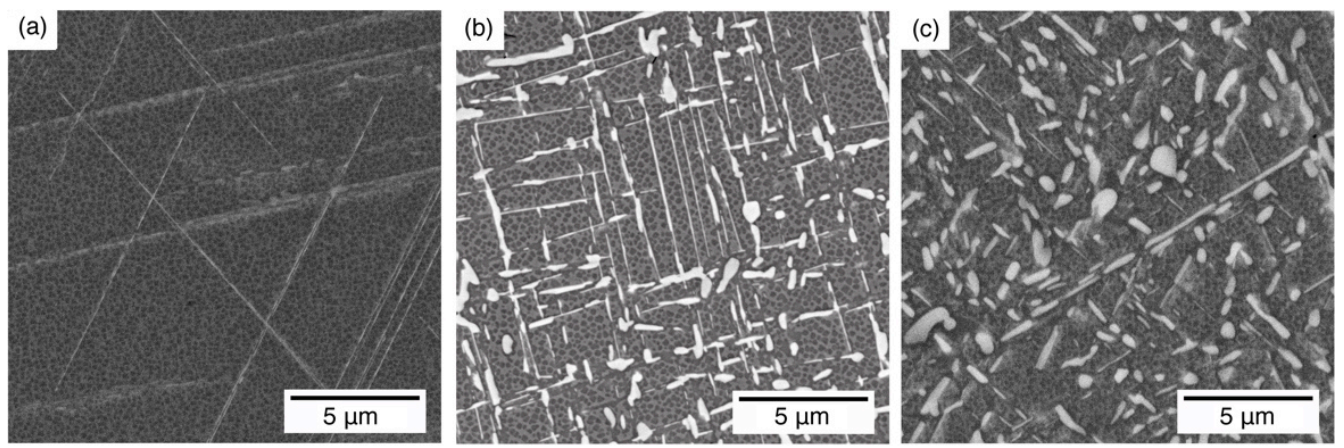

Fig. 9. BSE images of microstructure of 1.75 at.\% Mo alloy after thermal exposure at $800^{\circ} \mathrm{C}$ for (a) 175 , (b) 500 and (c) 1000 hours.

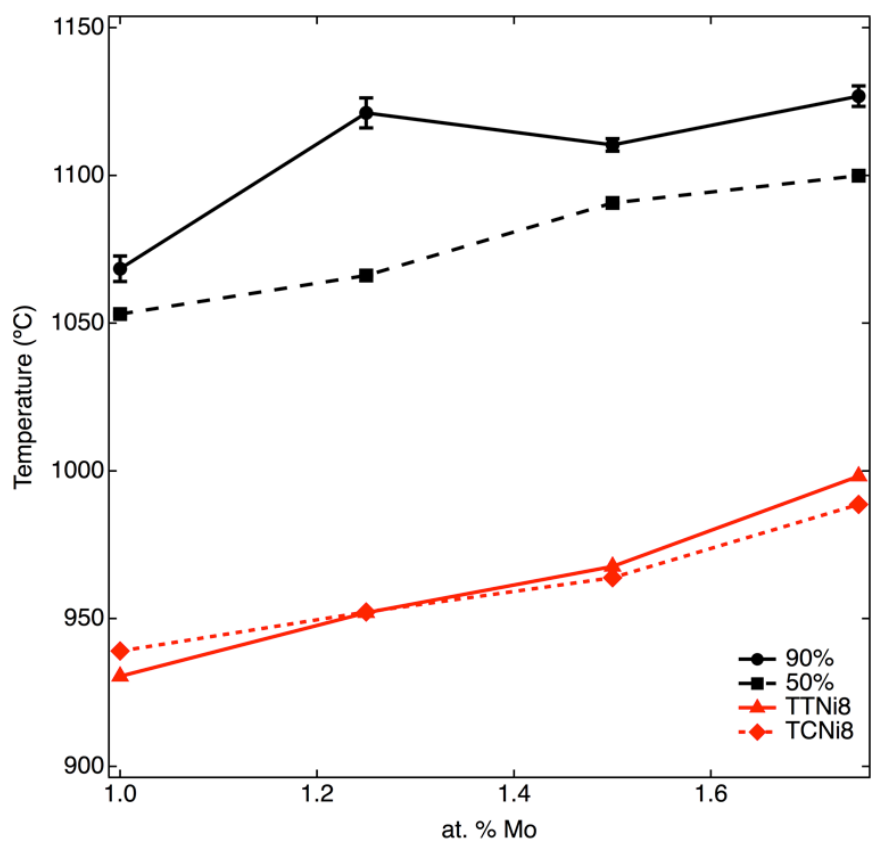

Fig. 10. Effect of Mo on experimental $\sigma$ solvus temperatures measured at $50 \%$ and $90 \%$ of the sigmoid and predictions made using databases, TTNi8 and TCNi8. 

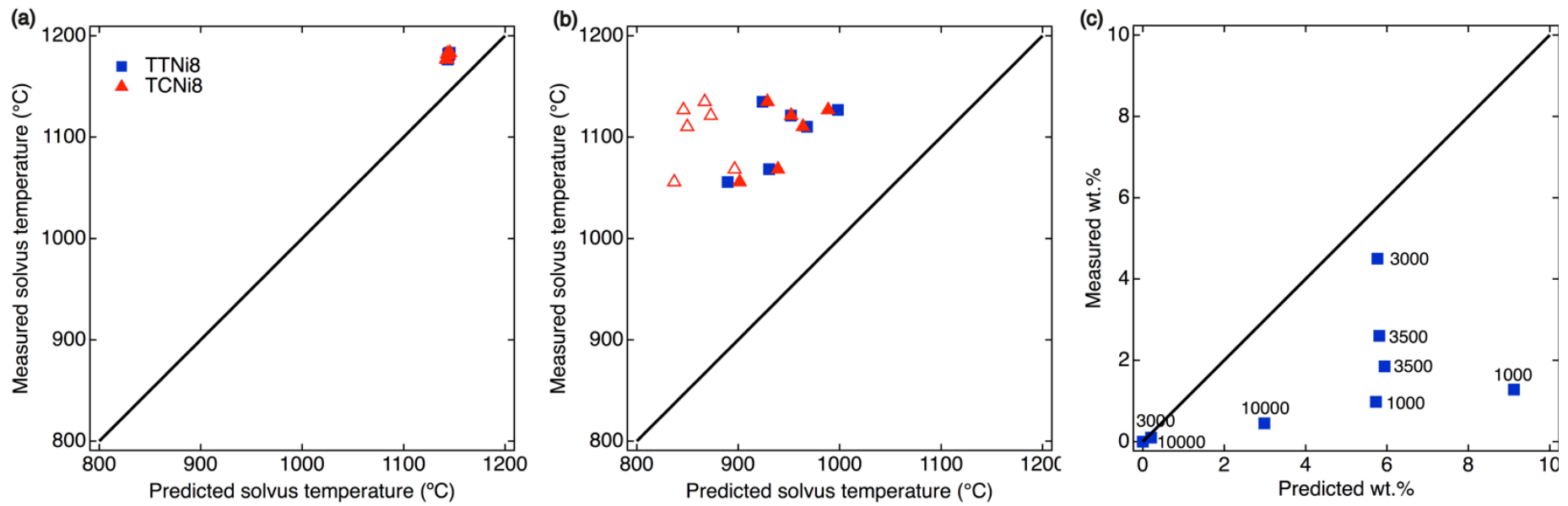

Fig. 11. Comparison of experimental and predicted data for (a) $\gamma^{\prime}$ and (b) $\sigma$ solvus temperatures from the current experiment, and (c) quantities of TCP phases with experimental data taken from the literature [24,27-29]. Labels on data points indicate the duration of the thermal exposure in hours. The outlined and filled triangles represent the prediction of two $\sigma$ phases of different compositions by the database TCNi8.

\section{Tables}

\begin{tabular}{|l|l|l|l|l|l|l|l|l|l|l|}
\hline & & Ni & Cr & Co & Mo & $\mathbf{W}$ & Nb & Al & Ti & Ta \\
\hline 1 Mo & Nominal & Bal. & 19 & 8 & 1 & 1 & 0.5 & 8 & 4 & 0.5 \\
\hline & Actual & Bal. & 19.16 & 7.99 & 1.06 & 1.05 & 0.50 & 7.80 & 3.82 & 0.49 \\
\hline 1.25 Mo & Nominal & Bal. & 19 & 8 & 1.25 & 1 & 0.5 & 8 & 4 & 0.5 \\
\hline & Actual & Bal. & 19.19 & 7.99 & 1.25 & 1.03 & 0.50 & 7.76 & 3.93 & 0.49 \\
\hline $\mathbf{1 . 5}$ Mo & Nominal & Bal. & 19 & 8 & 1.5 & 1 & 0.5 & 8 & 4 & 0.5 \\
\hline & Actual & Bal. & 18.95 & 7.90 & 1.56 & 1.01 & 0.50 & 7.72 & 4.03 & 0.46 \\
\hline 1.75 Mo & Nominal & Bal. & 19 & 8 & 1.75 & 1 & 0.5 & 8 & 4 & 0.5 \\
\hline & Actual & Bal. & 19.14 & 7.94 & 1.74 & 0.99 & 0.50 & 7.74 & 4.17 & 0.46 \\
\hline Alloy A & Nominal & Bal. & 18.5 & 8 & 1.25 & 1 & 0.5 & 7.5 & 4 & 0.5 \\
\hline & Actual & Bal. & 18.36 & 7.84 & 1.23 & 1.01 & 0.50 & 7.22 & 4.13 & 0.46 \\
\hline Alloy B & Nominal & Bal. & 18.5 & 8 & 1.25 & 1 & 0.5 & 8 & 4 & 0.5 \\
\hline & Actual & Bal. & 18.59 & 7.98 & 1.26 & 1.04 & 0.49 & 7.75 & 3.9 & 0.48 \\
\hline
\end{tabular}

Table 1. Nominal compositions of the alloys alongside their actual compositions in at. $\%$ as measured using EPMA. 